\title{
APEX, the Astrophysical Plasmadynamic EXplorer: An EUV High Resolution Spectroscopic Observatory
}

\author{
M Kowalski \\ Naval Research Laboratory, Code 7655.3, Washington, DC 20375
}

\begin{abstract}
APEX is a proposed Small Explorer satellite that will obtain highresolution EUV spectra of white dwarfs, CVs, stellar coronae, and the local ISM. The APEX effective area $\left(30-50 \mathrm{~cm}^{2}\right)$ and resolution $(\sim 10,000)$ are an order of magnitude improvement over Chandra.
\end{abstract}

APEX, a proposed mission Small Explorer mission, will investigate the density, temperature, composition, magnetic field, structure, and dynamics of hot astrophysical plasmas $(\log \mathrm{T}=5-7)$, which emit the bulk of their radiation at EUV wavelengths and produce critical spectral diagnostics not found at other wavelengths. With an effective area $1 \mathrm{~cm}^{2}$ and resolution $\mathrm{R} \sim 400$, EUVE moved astronomical spectroscopy into the extreme ultraviolet. Chandra and XMMNewton have demonstrated the promise of high-resolution X-ray spectroscopy. The termination of EUVE has left a gap in spectral coverage at EUV wavelengths that CHIPS fills only partially, as it is optimized for diffuse emission and has only moderate spectral resolution $(\mathrm{R} \sim 150)$. We recently flew an APEX prototype (effective area $3 \mathrm{~cm}^{2}, \mathrm{R} \sim 3000$ ) on a NASA sounding rocket and successfully obtained the first high-resolution EUV spectrum of an astrophysical object, the white dwarf G191-B2B (Cruddace et al. 2002). However, even higher resolution and longer observations are needed to exploit the full range of plasma diagnostic techniques developed in laboratory and solar physics, with unambiguous line identification and measurement of line profiles and Doppler shifts. APEX spans the range $90-275 \AA$ with $R \sim 10,000\left(\Delta v=30 \mathrm{~km} \mathrm{~s}^{-1}\right)$ and effective area $30-50$ $\mathrm{cm}^{2}$. APEX complements the Chandra, FUSE, and CHIPS missions.

APEX addresses basic questions of stellar evolution and galactic structure through high-resolution EUV spectroscopy of white dwarf stars, CVs, the local ISM, and stellar coronae. The science goals are to answer such basic questions in understanding the behavior of million-degree plasmas in the Universe.:

PRIMARY SCIENCE GOALS: (1) What is the evolutionary history of white dwarfs? (2) What are the density, temperature, ionization state, and depletion level of the local ISM? (3) Where are the emission sites in CVs, and what are their temperature, density, composition, and dynamics?

SECONDARY SCIENCE GOALS: (4) What are the structure and dynamics of stellar coronae? (5) What causes the features in the stellar differential EM distributions? (6) What is the relationship between coronal heating and flares? (7) What is the relative importance of magnetic reconnection and MHD and acoustic waves in coronal heating? (8) How do coronae evolve over stellar lifetimes? (9) How do coronal abundances differ from the photospheric abundances? 


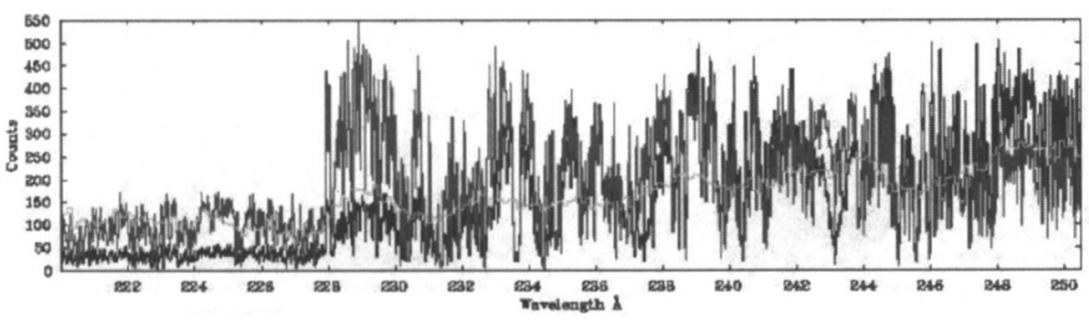

Figure 1. Simulated 4 ksec APEX exposure of the DA white dwarf Feige 24 for $\mathrm{H}$ layer masses of $10^{-13}$ (gray) and $10^{-14}$ (black) $M_{\odot}$. The white histogram shows the EUVE observation. Poisson noise has been added, but all fluctuations are real spectral features.

We illustrate APEX capabilities for the first goal (Fig. 1). The rapid post main sequence evolution of white dwarfs into two main types, H-rich DA and Herich DO and DB stars, is complicated and poorly understood. Composition is determined by core nucleosynthesis, shell-burning, and stellar mass-loss. Measured abundances should reflect their evolutionary paths. However, observed patterns may be affected also by gravitational settling (diffusion), selective radiative levitation, magnetic fields, and interstellar accretion. $T_{\text {eff }}, \log \mathrm{g}$, and the interstellar environment influence the relative importance of these processes. Obtaining high-resolution EUV spectra of a diverse sample of white dwarfs is the key to understanding their evolution. Determination of the photospheric $\mathrm{He}$ content provides critical information. EUV spectroscopy of the He II Ly series (228-304 $\AA$ ) provides a factor of $\sim 100$ more sensitivity than the He II lines at FUV wavelengths. Significant quantities of heavy elements are also present in the hottest white dwarf atmospheres. Studies have begun to correlate abundance patterns with evolutionary status (Barstow et al. 2003), but trends are challenged by unexpected complexities in photospheric structure. New measurements of heavy element abundances at different atmospheric depths are required to resolve questions of the radiative levitation/diffusion balance and its effect on atmospheric structure. Lines are formed at different depths in the EUV and FUV, requiring abundance measurements in both bands. High-resolution FUV spectral data are available from IUE or HST, but EUVE lacked the spectral resolution provided by APEX to determine unique heavy element abundances.

APEX is a suite of 8 spectrometers (Fig, 2), each slitless and with a figured diffraction grating (focal length $3 \mathrm{~m}$ ) operating at near-normal incidence in a Wadsworth mount. Light enters each spectrometer through a collimated aperture, and is diffracted by a grating to form a focused spectrum of a chosen order onto a detector. All spectrometers are functionally identical, but have different wavebands defined primarily by the multilayer coating on each grating. The composite graphite structure has a diameter of $160 \mathrm{~cm}$ and a mass of $600 \mathrm{~kg}$. The instrument requires $400 \mathrm{~W}$ and produces $1.2 \mathrm{~Gb} /$ day of science data.

To obtain high sensitivity, APEX uses large diameter $(350 \mathrm{~mm})$ blazed ion-etched gratings, employs multilayer coatings to enhance efficiency (Kowalski et al. 2003), and records spectra with efficient (alkali-halide photocathode) 

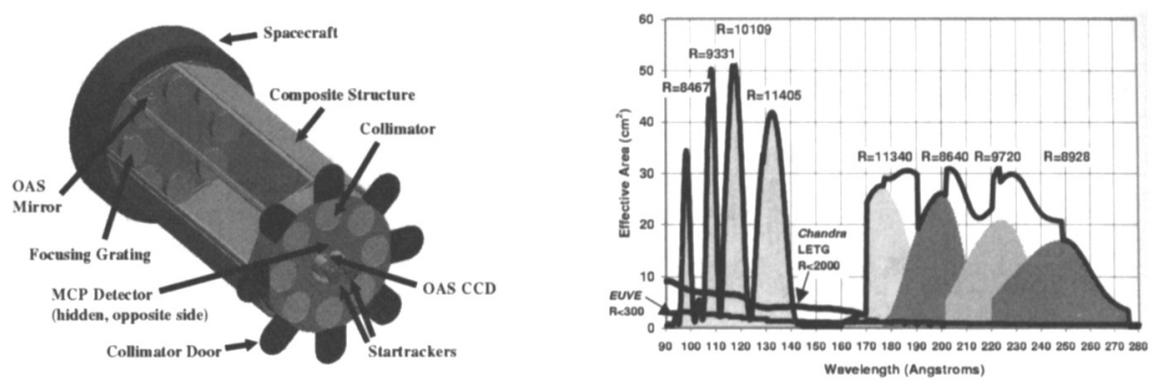

Figure 2. APEX payload. Figure 3. APEX predicted performance.

photon-counting microchannel plate (MCP) detectors with high spatial resolution (15 microns). Unlike FUSE, APEX has no slit and thus requires only modest pointing $\left(\sim 1^{\prime}\right)$, but accurate aspect knowledge and measurement of instrument flexure are required for high spectral resolution. Two startrackers produce precise $\left(<1^{\prime \prime}\right)$ pointing knowledge. APEX also employs two systems for measuring flexure, a pair of EUV telescopes and an optical alignment system (OAS), the latter providing continuous wavelength calibration for each spectrometer.

Predicted performance (effective area and $\mathrm{R}$ ) is shown in Fig. 3. In summary, APEX will achieve effective areas of $30-50 \mathrm{~cm}^{2}$ over the range $90-275 \AA$. At the location of important spectral lines the APEX effective area exceeds that of Chandra and EUVE by an order of magnitude. The APEX R 10,000 exceeds that of Chandra and EUVE by factors of 5 and 30, respectively.

The instrument is mounted on a 3-axis stabilized spacecraft. The Ball Aerospace and Technologies Corp. (BATC) RS-300 bus, suitably modified, meets the APEX requirements with adequate margins. The payload is launched by a Taurus vehicle, and payload size and weight fit comfortably within limits for the 2210 fairing. APEX is proposed for an August 2008 launch into a circular orbit with $500 \mathrm{~km}$ altitude and $28.5^{\circ}$ inclination. The baseline mission is 2 years. APEX will observe $\sim 100$ targets, which are selected carefully to maximize science, and all have been detected in the EUVE and ROSAT WFC surveys. The mission is a collaboration between the Naval Research Lab., the Lawrence Livermore National Lab., the University of Leicester, the University of California Berkeley, and a consortium of institutions that support the science data analysis.

\section{References}

Barstow, M. A., et al. 2003, MNRAS, 341, 870

Cruddace, R. G., et al. 2002, ApJ, 565, L47

Kowalski, M. P., et al. 2003, Proc. SPIE, 5168, 21 